\title{
Literature Review of Mobile Applications Testing on Cloud from Information Security Perspective
}

\author{
Shilpa Bahl*1 \\ CSE/IT KIIT College Of Engineering, Gurgaon \\ gerashilpa@gmail.com
}

\author{
M.M Chaturvedi ${ }^{2}$ \\ SET, Ansal University \\ Sector-55, Gurgaon \\ mmchaturvedi@ansaluniversity.edu.in
}

\begin{abstract}
The adoption of mobile application is increasing at enormous rate due to their improved functionality and features. Increased storage and computing power has augmented its utility. However, these mobile applications are still intrinsically limited by a relative lack of bandwidth, computing power, storage and energy compared to desktops. To overcome these limitations, the concept of Mobile Cloud Computing (MCC) providing abundant computing power and sufficient storage space besides large infrastructure has evolved. Cloud computing has its impact on all the stages of Software life cycle, including testing of mobile devices. In this paper we have reviewed Testing as a Service (TaaS) provided by Cloud computing. Testing of mobile application is more complex and time consuming as compared to traditional desktop applications.

This paper reviews some published results in two major research fields ie cloud computing and TaaS and discusses architecture of cloud computing and TaaS in terms of necessity, features, emerging trends, benefits and gaps while focussing on security and privacy issues for mobile application. When we run our applications on the cloud, we are sharing our critical data with cloud and, therefore, security and privacy of data is a very serious issue to be considered.
\end{abstract}

\section{Keywords}

Cloud Computing, Mobile Cloud Computing (MCC), TaaS, Information Security and Privacy.

\section{INTRODUCTION}

\subsection{Cloud Computing}

The objective of cloud computing is to expand test capabilities and capacity giving standardized approach to testing by providing automated tools on cloud and utilizing cloud services on the fly in pay-as-you-go manner without investing in new infrastructure, licensing new software, and training new recruits. The Service and Deployment models of cloud are shown in Fig 1 .

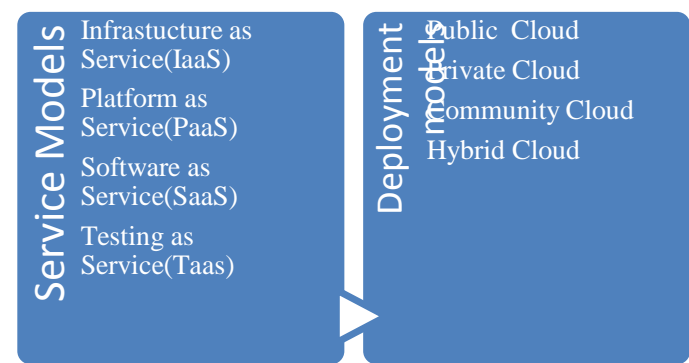

Fig:1 Cloud Computing Models

Cloud computing has a new identity by the developments in virtualization, distributed computing, utility computing, web and software services technologies [23].According to Kiah [13] the cloud has a layered architecture in which it has a backbone layer (physical servers and switches), supervisor software layer (OS, Hypervisor, Middle-ware),Software infrastructure layer shown in fig 2 which provides data storage, communication as service and infrastructure as services .

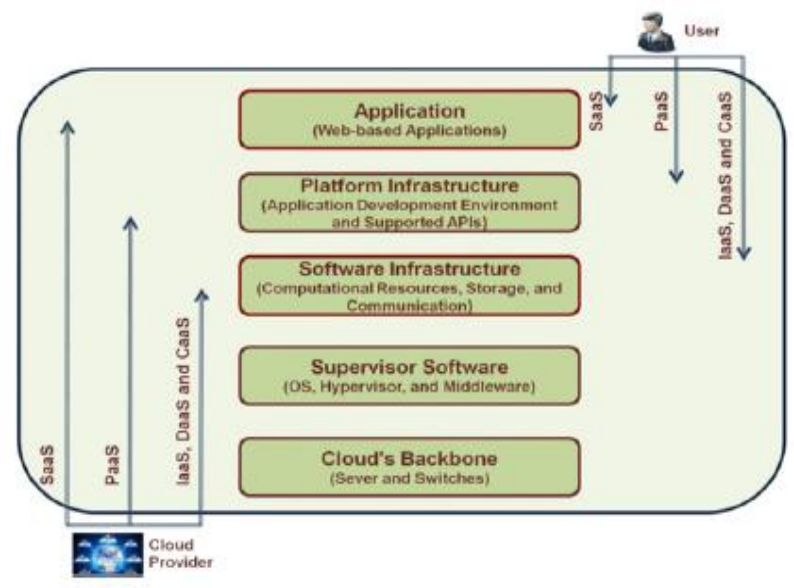

Fig:2 Layered Architecture of Cloud Computing [2]

The platform infrastructure layer provides an application development platform and a set of Application Programming Interfaces (APIs) for the developers for example Google App engine. The topmost application layer in the fig 2 allows users to access and use applications installed on a cloud provider's data centre through the Internet. Cloud computing provide a new ground of a computing paradigm for resource constrained mobile devices called MCC (Mobile Cloud Computing).

Cloud also consists of a large infrastructure (hardware, processors, hard disks, memories) so there may be a possibility of failure, there must be some critical recovery strategies. It is very crucial to test these recovery software's and strategies. Gunwai [29] has discussed two new recovery strategies for cloud FATE (Failure testing Service) and DESTINI (Declarative testing specification) the former test cloud system 
for multiple failure scenarios and the later is implemented for clear and precise specifications of recovery procedures

\subsection{Concept of MCC}

The term "mobile cloud computing", evolved long after "cloud computing. Mobile devices being battery powered, have limited processing power, low storage, less security, unpredictable Internet connectivity, and less energy. To augment the capability, capacity and battery time of the mobile devices, computationally intensive and storage demanding jobs should be moved to cloud .Therefore according to Kiah [13] MCC is defined as " a service that allows resource constrained mobile users to adaptively adjust processing and storage capabilities by transparently partitioning and offloading the computationally intensive and storage demanding jobs on traditional cloud resources by providing ubiquitous wireless access",

Limitations with MCC can be data replication, consistency, limited scalability, unreliability, unreliable availability of cloud resources, portability, trust, security, and privacy. This paper will focus on information security issues for mobile applications. Due to resource limitation, the security algorithms proposed for the cloud computing environment cannot be directly run on a mobile device. There is a need for a lightweight secure framework that provides security with minimum communication and processing overhead on mobile devices. In section 2 we will discuss automated mobile application testing, testing as service provided on cloud for mobile applications and in section 3 will focus on various security issues which are taken into consideration while testing a mobile application on cloud. Section 4 will give a brief of various techniques and tools available on cloud for testing of mobile applications.

Chonho Lee has discussed in his survey the applications[6] of MCC, mobile commerce, mobile learning, mobile healthcare ,mobile gaming and some general applications include sharing photos and video clips efficiently and tagging friends in popular social networks as Twitter and Face book, shopping ,travelling, showing maps, searching information(locations, images, videos, voices etc), to detect traffic lights for the blind and a cloud computing framework to monitor different corners in a house through a mobile device.

\section{LITERATURE SURVEY}

According to the survey Cloud computing includes various technologies "Distributed computing", "Parallel computing" and "Grid computing". It emerged in autumn 2006 by Google engineer Christopher responsible for "Google 101"project after that IBM has a joint Project with Google on "Cloud" and afterwards many companies launched various plans on "Cloud computing" emerged as the synonym for the next generation internet revolution. In the recent Years the major IT giants for different cloud service providers are Amazon cloud for storage, Google cloud for development, Trend micro cloud for safety and security. Among the 10 listed major strategies of Information Technology the "Cloud computing" is still the best. According to a recent study by ABI Research, a New York-based firm, more than 240 million businesses will use cloud services through mobile devices by 2015 .

Cloud computing era provides (EaaS) everything as a service; storage, resources, computing resources, development environment, testing, security etc. Many formal definitions have been proposed in both academia and industry, the one provided by U.S. NIST (National Institute of Standards and Technology).
Cloud computing is a model for enabling convenient, ondemand network access to a shared pool of configurable computing resources (e.g., networks, servers, storage, applications, and services) that can be rapidly provisioned and released with minimal management effort or service provider interaction [33].

Though there is a concept of SLA (Service level agreement) both from the users perspective not only for ensured provision of system functionalities and performance at reasonable cost but also for fault tolerance and recovery capabilities in reaction to failures.

Cloud computing is increasingly becoming the means through which online services are made available. V.Balasubramanian [19] has discussed various service models of cloud computing focussing on Taas model, its architecture various service components and the key features of the TaaS architecture. Chonho Lee[6] has discussed more about Mobile cloud computing architecture and applications and also various issues related to the cloud environment, security and the privacy of the data and the performance. The definition, Scope, importance and the mobile testing process on cloud and different approaches with comparison to conventional mobile testing approaches is enlightened by Jerry Gao [7]. The advantages discussed includes lesser test environment cost due to multiple virtual machines ( $\mathrm{VM}$ ) for test environments on one host server, isolated VM so that if one VM crashes would not affect the other VM's and stored VM image files that can be freely copied or moved.

Riungu and Taipale [35] discussed that testing of software's on cloud is influenced by conditions such as level of domain knowledge needed to test an application, flexible and cost effective, security and economy of scale and cloud computing as a booming paradigm and the software testers to polish their skills. According to Vengattaraman [30] in cloud environment for software testing there are number of clouds having different testing techniques. So it is the responsibility of service manager to supervise and co-ordinate between the core activities of respective layered entities of overall cloud environment and define the set of required clouds depending on the testing techniques required by any product of the client.

What is the importance of cloud based testing of mobile applications and what are the various types of CBT are discussed by Mohata [15]. Cloud testing which offers a shared test environment so that user need not to set up and maintain various testing platforms to ensure website or mobile application portability and compatibility and other issues like functionality ,interoperability, performance etc.Test scripts are recorded by the user from the local browser or with any of the OS in case of a mobile application .The scripts are then submitted to the cloud for testing to be executed automatically with the latest testing tools available there. Though cloud testing is providing a large number of static and dynamic testing and other services but applications hosted on remote clouds have lower controllability, uncertainties and observability as compared to conventional in house hosted applications. Automation has made an accountable impact in the testing field is discussed by Fuyang Peng[31] i.e. implantation and architecture of cloud based automatic software test environment (CASTE).To test a software on cloud it has two conditions ie the system under test is accessible online and testing infrastructure is hosted in the cloud.The automated testing environment is available all the time round the clock as the major advantage.

Testing has an endless impact on software's, hardware's and networks as well. A lot of work is done in this area and a lot more to go, if we emphasize on software testing of mobile applications, Muccini[16] discussed that earlier traditional 
approach was followed. Now a day's automated testing is playing a vital role to test mobile applications to overcome the challenges as it is different from desktop testing. Mobile application testing needs different and specialized techniques. Some of the respective peculiarities[16] and implications of testing for apps4mobile (traditional apps rewritten to run on mobile devices) and mobile apps (that make use of contextual information to generate context based outputs) eg mobile connectivity needs test for reliability performance etc, autonomy needs monitoring of energy consumption, adaptation needs test for adaptation correctness, diversity of phones and OS needs compatibility and OS testing ,new PL needs white box and black box testing and most important now a days for touch screen needs touch screen response and usability testing. The major area of concern for mobile application testing is the need of testing automation towards all layers and the layers among the application, application frame work, OS, and hardware layers. The most feasible and cost effective solution for automated mobile application testing is outsourcing cloud and crow-based testing which also provides specialized testing skills and laboratories to make thorough testing of critical mobile applications affordable. According to Starov \& vilkomir[32] there are TaaS solutions in mobile development as they have proposed CTMOS(Cloud Testing of mobile systems)with core infrastructure that adds all core testing functionalities, non functional testing, test case generation and test plan approaches jointly implemented in one platform over the cloud .It also provides possibility of majorly all use cases. Two Research attempts are discussed by Terner and Konstantinidis [21] and [34]respectively for creating and investigating test-bed cloud solutions for mobile development ie ATTACK(Android Tactical Application Assessment and Knowledge) cloud and Smart lab respectively both are distributed systems that connect a set of mobile devices having android OS for application investigation, development and testing.

When we are putting such critical information in hands of a third party other than our own testing team and development team, the most challenging issue is the security and privacy of the mobile applications. A lot of research is going on the security issues of mobile application testing on cloud. Markov [41] proposed various software testing models against information security for the assessment of technical software security at different stages of its life cycle. The classification of models will help in making the right decision to choose a respective model based on the available statistics, and the results of the models application is helpful for justifying the testing labour cost and reporting records which may be helpful for customer to understand the reliability of the model. Testing of mobile application against security has also being automated as discussed by Gilbert [36]. The overall summary is described in a tabular form for a quick review in table 1

Table:1 Review of Testing of Mobileapps on cloud

\begin{tabular}{|l|l|l|}
\hline Author & $\begin{array}{l}\text { Area } \\
\text { Discussed }\end{array}$ & Proposed Work(Year) \\
\hline $\begin{array}{l}\text { V.Balasubr } \\
\text { amanian, } \\
\text { Chohno } \\
\text { Lee, Jerry } \\
\text { Gao }\end{array}$ & $\begin{array}{l}\text { Cloud } \\
\text { Computing \& } \\
\text { mobile cloud } \\
\text { computing } \\
\text { Introduction }\end{array}$ & $\begin{array}{l}\text { CC is Online service. } \\
\text { Provides Everything as } \\
\text { a Service(EaaS). } \\
\text { Defination, Scope, } \\
\text { importance and the } \\
\text { Mobile testing process } \\
\text { on cloud. } \\
\text { Includes lesser test } \\
\text { environment cost due to }\end{array}$ \\
\hline
\end{tabular}

\begin{tabular}{|c|c|c|}
\hline & & $\begin{array}{l}\text { multiple virtual } \\
\text { machines (VM) for test } \\
\text { environments on one } \\
\text { host server and isolated } \\
\text { VM. } \\
\text { Discussed various } \\
\text { service models under } \\
\text { CC. } \\
\text { Focussed on TaaS } \\
\text { Architecture }\end{array}$ \\
\hline $\begin{array}{l}\text { Riungu and } \\
\text { Taipale }\end{array}$ & $\begin{array}{l}\text { Cloud Based } \\
\text { Testing of } \\
\text { Software's }\end{array}$ & $\begin{array}{l}\text { Conditions by which } \\
\text { testing of software's on } \\
\text { cloud is influenced. }\end{array}$ \\
\hline $\begin{array}{l}\text { Vengattara } \\
\text { man }\end{array}$ & $\begin{array}{l}\text { Software } \\
\text { testing } \\
\text { techniques on } \\
\text { provided by } \\
\text { different } \\
\text { clouds }\end{array}$ & $\begin{array}{l}\text { Different cloud provide } \\
\text { different testing } \\
\text { techniques according to } \\
\text { the requirement of the } \\
\text { client. } \\
\text { The responsibility of } \\
\text { service manager to } \\
\text { coordinate between } \\
\text { client and cloud. }\end{array}$ \\
\hline Mohata & $\begin{array}{l}\text { Cloud Based } \\
\text { Testing Of } \\
\text { Mobile } \\
\text { Applications }\end{array}$ & $\begin{array}{l}\text { Discussed Cloud Based } \\
\text { Testing(CBT)of mobile } \\
\text { applications. } \\
\text { Offers a shared test } \\
\text { environment. } \\
\text { Types of CBT }\end{array}$ \\
\hline $\begin{array}{l}\text { Fuyang } \\
\text { Peng }\end{array}$ & $\begin{array}{l}\text { Automated } \\
\text { Testing of } \\
\text { Mobileapps. }\end{array}$ & $\begin{array}{l}\text { Test scripts are made on } \\
\text { local browser or any OS } \\
\text { and submitted to the } \\
\text { cloud for automated } \\
\text { testing. }\end{array}$ \\
\hline Muccini & $\begin{array}{l}\text { Peculiarities } \\
\text { and } \\
\text { implications } \\
\text { of mobile } \\
\text { application } \\
\text { testing on } \\
\text { cloud }\end{array}$ & $\begin{array}{l}\text { Mobile application } \\
\text { testing is different from } \\
\text { traditional desktop } \\
\text { testing. } \\
\text { Respective peculiarities } \\
\text { and implications for } \\
\text { Apps4mobile and } \\
\text { mobile Apps } \\
\text { The need of testing } \\
\text { automation towards all } \\
\text { layers i.e. application, } \\
\text { application frame work, } \\
\text { OS, and hardware } \\
\text { layers. } \\
\text { Outsourcing the testing } \\
\text { on cloud or by crow- } \\
\text { based solution is cost } \\
\text { effective and feasible. }\end{array}$ \\
\hline $\begin{array}{l}\text { Starov \& } \\
\text { vilkomir, } \\
\text { Turner \& } \\
\text { Konstantini } \\
\text { dis }\end{array}$ & $\begin{array}{l}\text { CTMOS(Clou } \\
\text { d Testing of } \\
\text { Mobile } \\
\text { Systems),ATT } \\
\text { ACK,SmartLa } \\
\text { b }\end{array}$ & $\begin{array}{l}\text { Adds all core testing } \\
\text { functionalities, non } \\
\text { functional testing, test } \\
\text { case generation and test } \\
\text { plan approaches jointly } \\
\text { implemented in one } \\
\text { platform over the cloud. } \\
\text { Discussed for Android } \\
\text { OS creating and } \\
\text { investigating test-bed } \\
\text { cloud solutions for } \\
\text { mobile development ie } \\
\text { ATTACK(Android) } \\
\text { cloud and Smart lab } \\
\text { respectively both are } \\
\text { distributed systems. }\end{array}$ \\
\hline
\end{tabular}




\begin{tabular}{|c|c|c|}
\hline $\begin{array}{l}\text { Markov, } \\
\text { Gilbert }\end{array}$ & $\begin{array}{l}\text { Security \& } \\
\text { privacy issues } \\
\text { of testing } \\
\text { Mobile } \\
\text { applications } \\
\text { on cloud }\end{array}$ & $\begin{array}{l}\text { Software testing models } \\
\text { against information } \\
\text { security implemented at } \\
\text { different stages of its } \\
\text { life cycle. } \\
\text { Threat detection: } \\
\text { CloudAV } \\
\text { Information Security: } \\
\text { K-Anonymity. } \\
\text { Challenges: } \\
\text { Dependency on the } \\
\text { Internet, } \\
\text { Information security and } \\
\text { testing at all layers. } \\
\text { Automation of testing of } \\
\text { mobile application is } \\
\text { also discussed. }\end{array}$ \\
\hline Selvam & $\begin{array}{l}\text { Testing tools } \\
\text { and } \\
\text { Techniques }\end{array}$ & $\begin{array}{l}\text { Android: JUnit. } \\
\text { J2ME:J2MEUnit } \\
\text { Windowsphone:SilverL } \\
\text { ightby Microsoft } \\
\text { Monkeyrunner Sikuli } \\
\text { and } \\
\text { Framework, } \\
\text { UI/Applicationexercise } \\
\text { r Monkey } \\
\text { Techniques:RMTS,App } \\
\text { inspector, }\end{array}$ \\
\hline
\end{tabular}

\section{MOBILE APPLICATION TESTING ON CLOUD}

According to Jerry Gao [7]Mobile Testing as a Service (known as Mobile TaaS) provides static and dynamic ondemand testing services for mobile applications and SaaS to support software validation and quality engineering processes by leveraging a cloud-based scalable mobile testing environment to assure pre-defined given service-levelagreements (SLAs). Mobile TaaS on clouds offers a new business model for diverse mobile software validation services using the pay-as-you-test model to achieve cost-sharing and cost-reduction in mobile computing resources, networks, cloud computing and storage infrastructures. Various types of testing levels provided by the cloud covers, unit testing, integration testing, User interface testing, regression testing, system testing and acceptance testing as shown in figure 3 . It also brief the test scope based on test levels ie. functional testing, performance, security, usability, Stress/load testing, localization testing and synchronization testing. The test environment shown in figure 3 covers either testing on real device and testing on device Emulators. Testing on real device can further be either an inhouse physical device testing or the focussed remote device connectivity which is cost effective and less time consuming for example Device Anywhere and Perfecto Mobile Test Cloud. Secondly the testing can be done using emulators again it is cost effective and covers a large range of devices .But the limitation with the later is that all the activities cannot be realistically emulated like taking a picture or a video and the device cannot be tested on different networks ie geo-location capability testing.

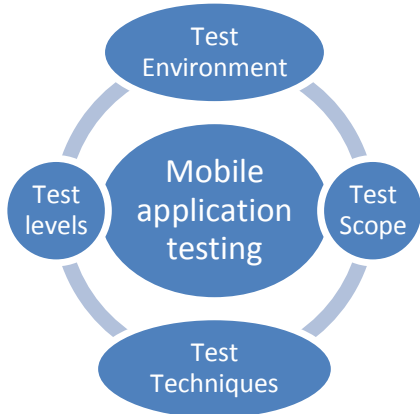

Figure:3 Mobile Application Testing Paradigm

Testing techniques may be manual testing, automated testing, risk based testing, scenario testing ,domain and Random testing techniques which are discussed in later sections.

Testing is resource intensive and the cloud offers these resource dynamically. Cloud based Testing use cloud infrastructure for software testing. Cloud offers certain benefits and challenges to testing. Some of the benefits of cloud testing are computational power, storage, automated tools and virtualization. Cloud testing provide two unique capabilities which cannot be found in traditional testing viz. virtualization (one can create customised test labs, almost same production environments and parallelize testing) and compatibility with generic cloud services (permitting use of public as well as private cloud)

\subsection{Architecture of TaaS Platform}

The user can utilize cloud platform by using any of the scenarios ie in the first one the user can upload the application on TaaS platform and can ask their own tester to test the application and can pay for the tools because the first layer is unified view so that they can directly reach out to TaaS platform through online or through help desk. It has both commercial an open source tools. where the services and the tools are bundled and the customer can use any of them by first getting registered with Taas platform In the second scenario the customer can upload the application on the cloud and ask the other testing company to test the application and pay for the usage . For that in the second layer is the customer need to sign in SLA which is an agreement between customer and the company. In the third layer virtualization takes place which is like managing our physical system virtually and the fourth layer is cloud adapter as Taas platform is integrated in the cloud with these adapters so that Taas platforms can be integrated by other providers also [19].

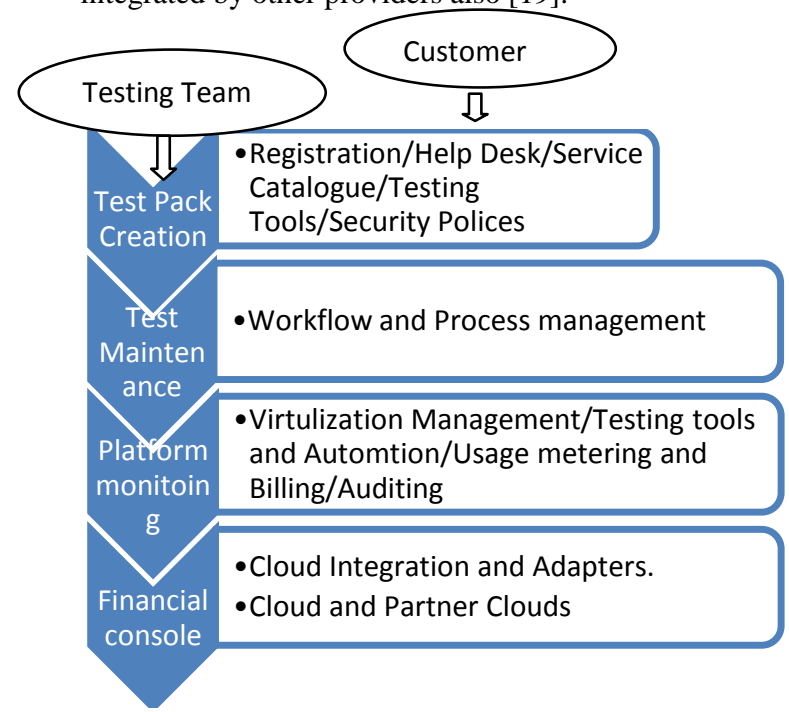

Fig 4: Architecture of TaaS Platform [19] 


\subsection{Features of Cloud Based Testing}

The main features of TaaS are collectively explained as it provides diverse services to their end users and customers with well-defined service-level-agreements, price models and utility billing as the basic parts and other services viz. testing as a service large-scale cloud-based data traffic simulation [8] and tracking and monitoring service, which allows test engineers to track and monitor diverse program behaviours at different levels of execution for the testing purpose.

Some basic features that must be provided by the cloud for TaaS model are availability of services anytime, authentication, digital rights management, enhanced data access efficiency, provision of context-aware mobile cloud services, network access management, quality of services (clone cloud, cloudlets), pricing and service convergence 3.3Different views of Cloud based Testing According to Jerry Gao [8] there are different views of cloud based testing:

- Vendors' view which present the view from the engineers who had actually constructed the cloud.

- User Oriented view who conducts test for the QA and the capabilities of the cloud.

- The clients or the users whose applications have to be run on the cloud and interaction of those applications with each other. The testing is done to assure the quality of the cloud-based application systems over clouds as described in Fig 5[8]

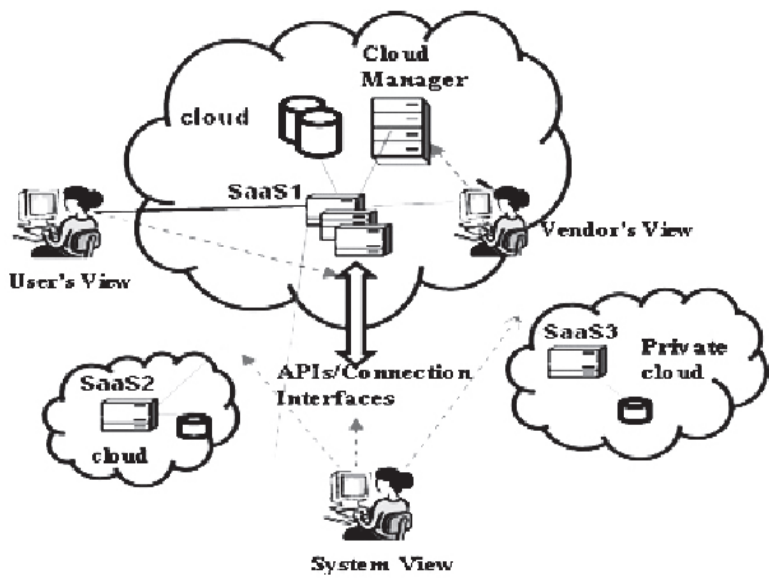

Fig 5:- Views of Cloud Based Testing [8]

\subsection{Need of testing on cloud}

Testing new software often requires costly servers, storage and network devices only for a limited time [7]. These computing resources are either not used or underutilized after testing, thus result in waste of precious resources. Mobile application providers have to maintain the quality of their services over various combinations of platforms. The computing platforms may encompass various browser technologies with different backend support running on various mobile OS. To ensure a reliable service, providers have to test their services on all these platforms. In addition to this testing of mobile applications, software's also need test automation tools which can also be easily migrated to cloud.

One more reason for the adoption of cloud computing is an economy of scale i.e. it provides a pay-per-use type of service, thus reducing the upfront investment in some cases; for example using testing tools when needed and using various platforms for running any application without investing into every platform.

Another aspect of cloud testing is assured availability without undue delays and from a reliable source [15].It also maintains the integrity of the data.

\section{FOCUSSED SECURITY ISSUES DURING TESTING MCC}

In MCC it is the major responsibility of the cloud provider to protect user privacy and data/application secrecy. Some of the security issues for mobile users and for data are discussed by Hoang T. Dinh [6] as mobile users are more exposed to attacks like malicious codes (e.g., virus, worm, and Trojan horses) and vulnerability eg. Global Positioning System (GPS) enabled phones have some critical privacy issues. The area of concern is how can we assure the security of cloud based application processes and business data inside a third-party cloud infrastructure [25]? What are the test models, test techniques and tools for security testing for end-to-end applications in/on/over clouds? How can we assure and assess user privacy in a cloud infrastructure [1]. As it is impossible to run virus detection software always on mobile devices due to resource limitations, so we can move these threat detection capabilities to cloud for example Cloud AV [6] which provide in-cloud service for Malware detection and file verification ,in addition to this it not only enhance the efficiency of detecting malware, but also improves battery lifetime up to $30 \%$.Its host agent and network service components [18], [17] are lightweight process that runs on mobile devices, and its function is to inspect the file activity on a system. Therefore, by moving these capabilities to cloud we can also use multiple antivirus engines in parallel by hosting them in virtualized containers. On the other hand for privacy issues which is faced by the mobile users having GPS and Location Based Services(LBS) enabled phones, providing there private information like their current location. Location trusted server (LTS)[27] is used to address this issue. As shown inFigure6

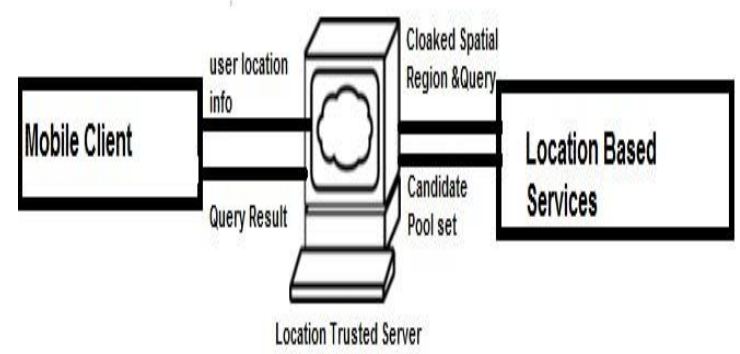

Fig 6: Mobile security service setup on the cloud

After receiving mobile users' requests, LTS gathers their location information in a certain area and cloaks the information called "cloaked region" based on a "k-anonymity" concept [20] to conceal user's information. The "cloaked region" is sent to LBS, so LBS know only general information about the users but cannot identify them [24].Point out the problem in case LTS reveals users' information, or if LTS colludes with LBS, users' information will be in danger. "Cloaked region" is generated on mobile devices based on Casper cloaking algorithm [4]. 


\subsection{A Survey on security framework for MCC}

When we talk about the security it should have following two main perspectives:

\section{- Security of data}

Itani [11] proposed an energy efficient integrity verification scheme for mobile clients which verifies the integrity of the file stored on the cloud server using an incremental message authentication code.

Jia et al proposed[12] outsourcing data and security management on cloud by proxy re-encryption and identity based encryption schemes.

Hsueh proposed a scheme[9] for smart phones for security, integrity and authentication of mobile user data by storing the traditionally encrypted files with user credentials hosted by an adversary who can utilize user credentials to impersonate the user later on.

Yang et al [29] proposed a scheme for resource constrained mobile phones for TTP is responsible for handling encoding/decoding, encryption/decryption, signature generation and verification on behalf of mobile user.

Zhou and Huang [28] proposed a scheme which offloads the processing and storage intensive encryption and decryption operations on the cloud without revealing any information about the data contents and the security keys.

\section{- Security of Applications}

Zang et al.[26] proposed a scheme an elastic mobile application model and covered the security aspects of the proposed model. The proposed security model deals with the secure installation of elastic application, authentication among web lets, secure migration of web lets, and authorization of web lets while using external web services. The proposed model safeguards the modification of a web let only at installation time with the help of a developer signed hash value In [5], Xiao and Gong proposed a scheme to generate automatic dynamic credentials with the mutual coordination of mobile devices and cloud. The generated credentials are used to protect the mobile user from different type of attacks. The proposed scheme considers cloud as a fully trusted component to implement the solution accurately.

In [10], Chow et al. proposed a policy based cloud authentication platform that addresses the client device authentication issue using implicit authentication. To achieve privacy, mobile clients apply hash function on data (except GPS location) with a self-generated key and transfer the generated information to the data aggregator. An unusual pattern can be detectable without disclosing the noticeable user information. The mobile client has to apply a hash function on frequent generated information to achieve privacy.

In [29], Huang proposed secure data processing model for MobiCloud that provides enhanced security and privacy protection for mobile users with the help of multi-tenant secure data management, trust management. To provide strong security services to the user, the storage domain module and cloud trusted domain module are physically isolated. If the cloud trusted domain module is hosted by a trusted third party, there is an issue of scalability.

\subsection{Different forms of cloud based testing}

a)Testing of a cloud

Cloud environment should be tested and measured for their performance, availability, security capability and scalability in order to support efficient delivery of services. Cloud and SaaS vendors as well as end users are interested in carrying on this type of testing so it is from an external view

b) Testing inside a cloud

This is to check the quality of the cloud about the infrastructure and the capabilities of the cloud. Performed only by cloud vendors as only they have accesses to internal infrastructures and connections between its internal $\mathrm{SaaS}(\mathrm{s})$ and automatic capabilities, security, management and monitoring.

c)Testing over cloud

It test the cloud based applications over the cloud ,depending on the level of the application, service requirement and the specification over the public ,private and the hybrid clouds. It is used by the cloud-based application system providers

d)Testing a SaaS in a cloud

It test the quality of software's based on the cloud depending upon the functional and non functional requirements of the application

\subsection{Types of testing of mobile application on the cloud}

a)Load and Performance testing

Now a days we have majority of mobile application $s$ in Smartphone's which actually run on cloud for example marketplace, GPS applications on mobile, gaming etc therefore according to a survey by Cisco [3] as the traffic on internet increases in terms of number of users as well as the size of the data an application may get hits from all corners of the world .So in order to tackle all the users, the performance of the application needs to be measured in peak traffic.

\section{b) Functional Testing \& Browser Testing}

It test for the validation of the cloud environment, checks for the compatibility of different client interfaces and technologies for different platforms, OS and browsers. It also have to test for the proper functionality like for connectivity protocols and UI/client technologies inside a cloud.

c) Latency Testing Cloud testing is utilized to measure the latency between the action and the corresponding response for any application after deploying it in the cloud [15]

d) Governance Risk Compliance (GRC) testing. Its main focus is to find out threats, vulnerabilities and risks that are associated to all three parts of Cloud Computing Infrastructure as a Service (IaaS), Platform as a Service (PaaS) $\&$ Software as a Service (SaaS) and suggest the best practices to overcome it followed by many security agencies.

Some additional types testing can also be implemented depending upon the type of client interfaces ie fail over testing, capacity testing and Governance risk compliance testing Some of the other mobile testing types are given below in figure :7 


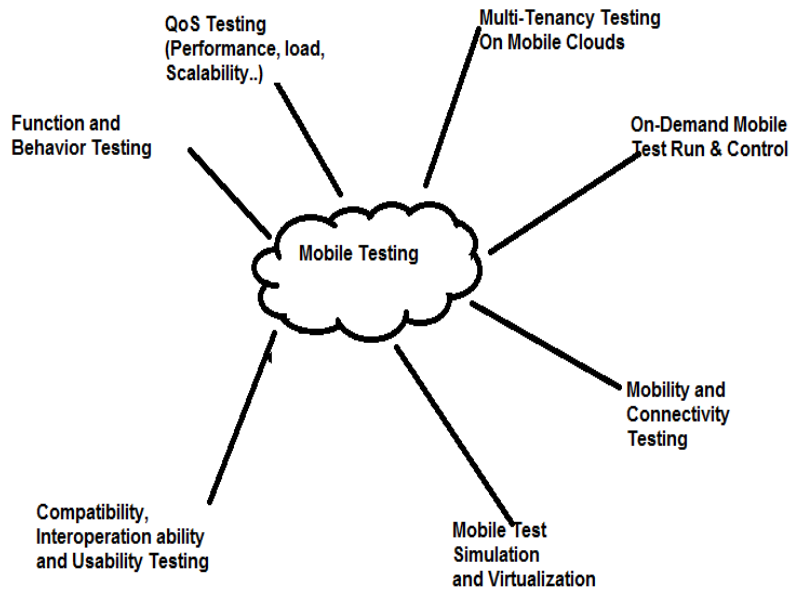

Fig: 7 Mobile application testing types

\subsection{Challenges of Cloud Based Testing of Mobile applications}

Some challenges of mobile application testing in cloud are as follows:

1. Dependency on the Internet-Applications is not installed locally in controlled environments. This become challenging for testers to duplicate the user environment.

2. Information Security- Since information travels through the Internet, testers have to perform testing against security on the communication medium to make sure there is no data leakage when data is sent over the Internet. Wiki leaks is an example of threats that we have to prepare for and test before the application can be released to customers.

3. Testing all layers - Testing the network connection, performance of the server, database, and software application adds multiple perspectives to testing. Further the test can be done for testing the communication between the layers, testing of the connected elements, and also plan for the risks.

\section{TOOLS AND TECHNIQUES FOR TESING MOBILEAPPS ON CLOUD}

A mobile software is first of all taken through compatibility testing ie the mobile application can run on various phones. But for the mobile vendors it is not feasible (Financially and on a short time constraint) to test for all the platforms and then launch the applications for a fast growing market. Therefore before announcing the application in the market it should be validated for the expected results.

Two approaches that can be followed other than manual testing is either by using device emulators which are available for corresponding popular mobile phones. But using emulators itself have drawbacks eg. it cannot cover all the aspects of real mobile (Screen sizes, real calling, network interoperability, updating of emulators due to new versions, emulators themselves have bugs).Some of the Automated tools used for different OS in smartphones are referenced by Selvam[39] e.g. for Android test cases can be developed by using invoking JUnit methods,for J2ME we use J2MEUnit ,for Windows phone 7 platform we use SilverLight Unit Test Framework Tweaked by Microsoft.

According to a survey by Hughes Systyque [40] there are multiple automated testing tools for mobile applications which can be implemented through cloud as well as on emulators. For different categories they are described as, for platform specific tools are INSTRUMENT and MONKEYRUNNER Generic script based tools are SIKULI and ROBOT FRAMEWORK, for random event generation tools are UI/APPLICATIONEXERCISER MONKEY and AUTOMATION, for white box testing tools are ANDROID INSTRUMENTATION and INSTRUMENTS and for black box testing tools are SIKULI and ROBOTIUM.

The other approach recently used is testing remote mobile systems on cloud which is discussed by Huang[37].RMTS is a mobile testing cloud through which an end user can request devices on demand to test their software with automated testing features.

Gilbert and Gon Chun[38] has discussed an automated security validation tool called appInspector which analyzes the applications and generates reports of potential security and privacy violations. At a higher level a master appinspector can create multiple appinspector nodes each including a virtual Smartphone. A brief overview of the architecture is given in figure 8 which shows the components of the system. Initially the appinspector installs and load the apps on virtual smartphone. The user input events are injected through input generator. Execution explorer traverse possible execution paths of the app to ensure thorough analysis. Information flow and action tracking components monitor flow of privacy sensitive information and generates logs. Appinspector also provides security analysis tools used after execution is completed to interpret the logs and generate a report as shown in figure 8 .

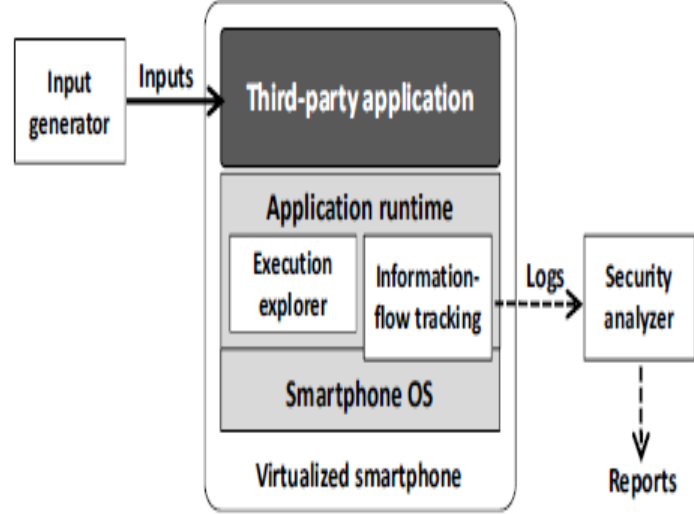

Fig:-8AppInspector Node Architecture [38]

The other approach recently used is testing remote mobile systems on cloud which is discussed by Huang[37].RMTS is a mobile testing cloud through which an end user can request devices on demand to test their software with automated testing features. That is the user can access a real mobile phone remotely and can operate them through web browser, including uploading,start-up, testing of mobile applications by clicking and swiping options. Automated testing scripts can be recorded and can be used to run on other mobile phones to improve testing efficiency. But RMTS has a two limitations firstly it is implemented only on Android platform and secondly only one user can use each mobile phone at a time which can be achieved by using the concept of virtualization which has yet to be implemented

\section{CONCLUSIONS AND FUTURE WORK}

Mobile Cloud computing combines the advantage of cloud computing and mobile computing, As cloud computing comprises of different service models ie SaaS, IaaS, PaaS,SaaS and TaaS from which Taas is the main focus of discussion in this paper and is a recent research field. This paper has tried to provide an overview of cloud computing, MCC ,features of Cloud computing, requirements, issues and challenges we face during testing of a mobile application on the cloud either on 
the real device or on device emulators. In the initial phase of testing device emulators can be used and end testing can be done on real devices on cloud. This paper has described the architecture of TaaS platform and its applications. This paper has also focussed on a very serious issue of TaaS ie information security of mobile devices on the cloud. As that is the main reason for user hesitaion to adopt mobile applications which run on cloud and store data on cloud. Some possible solutions given in the recent studies are also discussed. Various tools and techniques used for mobile application testing for diverse platforms are also listed with security as end objective. The test cases design can be focussed and categorized based on device specific features, network issues, mobile web browser, mobile OS specific features and mobile user location. Unit testing and functional testing covers 60 to $70 \%$ of the faults before installation of the application on device. Focussing on the security issues the paper has discussed an automated approach for security testing of android application on the cloud.

\subsection{Research agenda}

In future the various tools which are discussed in this paper can be enhanced to be implemented on various platforms because there are tools which are very platform specific or some tools are specific for a type of testing only ie either for Black box or for white box testing so they restrict our area of testing. As we have an advantage of availability of all types of tools present in the cloud but it will be more convenient if we have more generic tools for testing mobile applications. Still there are some tools which havenot yet used the concept of virtualization and thus hinder the usability for multiple users.

\section{OPEN ISSUES}

On the basis of the literature review, a number of open issues have been identified which are broadly categorized according to our area of interest. Firstly; the general information security issues eg. Intrusion detection and privacy preserving can be provided by knowledge of homogeneity attacks and background knowledge attacks. Secondly; the Mobile security can be ensured by providing authentication and biometrics or by K-Anonymity and encryption. Thirdly; the cloud can be secured in general by securing the firewalls by authentication , giving multiple firewalls for distributed networks, dynamically analyzing network traffic and detecting and correcting faulty networks.

\section{REFERENCES}

[1] AppLabs." Testing the Cloud". White paper, Internet:http://www.applabs.com/html/TestingtheCloud_7 86.html.

[2] Bahl Paramvir \& Richard Y. Han \& Li Erran Li \& Mahadev Satyanarayanan.2012. "Advancing the State of Mobile Cloud Computing”. Low Wood Bay, Lake District, UK. MCS'12.

[3] http://www.cisco.com/en/US/solutions/collateral/ns341/ns52 5/ns537/ns705/ns1175/Cloud_Index_White_Paper.pdf

[4]Chow C-Y \& Mokbel M. F \& Aref W. G,(2009).Query processing for location services without compromising privacy. ACM Transactions on Database Systems (TODS), vol. 34, no. 4.

[5] Chow. R\&J akobsson. M \& Masuoka.R \& Molina.J \& Niu.Y \& Shi.E\& Song.Z.(2010). Authentication in the clouds: a framework and its application to mobile users. Proceedings. ACM Cloud Computing Security Workshop, CCSW '10, Chicago, USA.
[6] Hoang T. Dinh \& Chonho Lee \& Dusit Niyato \& Ping Wang.A Survey of Mobile Cloud Computing: Architecture, Applications, and Approaches. Wireless Communications and Mobile Computing - Wiley.

[7] Jerry Gao, Ph.D. Professor \& W. T. Tsai, Ph.D. Professor, Mobile Testing-as-a-Service, [Power point Presentatio].25 ${ }^{\text {th }}$ June Accessed.

[8] Jerry Gao \& Xiaoying Bai \& Wei-Tek Tsai .(2011). Cloud Testing- Issues, Challenges, Needs and Practice . Software Engineering : An International Journal (SEIJ), Vol. 1, No. 1 .

[9] S.C. Hsueh \& J.Y. Lin \& M.Y. Lin.(2011) Secure cloud storage for conventional data archive of smart phones. Proceedings 15th IEEE Int. Symposium on Consumer Electronics, ISCE ' 11.

[10] D. Huang \& Z. Zhou \& L. Xu \& T. Xing \& Y. Zhong(2011)."Secure data processing framework for mobilecloud computing". Proc. IEEE INFOCOM Workshop on Cloud Computing, INFOCOM '11, Shanghai, China

[11] W. Itani \& A. Kayssi \& A. Chehab .(2010)."Energyefficient incremental integrity for securing storage in mobile cloud computing". Proceedings Int.Conference on Energy Aware Computing, ICEAC '10, Cairo, Egypt.

[12] W. Jia \& H. Zhu \& Z. Cao \& L. Wei \& X. Lin.(2011)."Asecure data service mechanism in mobile cloud computing", Proceedings IEEE Conference on Computer Communications Workshops, INFOCOM WKSHPS, Shanghai, China.

[13]Abdul Nasir Khana \& M.L. Mat Kiah \& Samee U. Khanb \& Sajjad A. Madanic.(2012)." Towards secure mobile cloud computing": A survey. Future Generation Computer Systems.08-003,Page no.1-5.

[14]A. Konstantinidis \& C. Costa \& G. Larkou \& D. Zeinalipour- Yazti.(2012).Demo: “A programming cloud of smartphones" .Proceedings of the 10th international conference on Mobile systems, applications, and services, ACM New York, NY, USA. pp. 465-466.

[15]Vinit B. Mohata1\& Dhananjay M.Dakhane \& Ravindra L.Pardhi.(2013). "Cloud Based Testing: Need of Testing in Cloud Platform"sInternational Journal of Application or Innovation in Engineering \& Management (IJAIEM) Volume 2, Issue 3 .

[16]Henry Muccini \& Antonio Di Francesco \& Patrizio Esposito."Software testing of mobile Applications Challenges and future Research Directions".

[17]J.Oberheide \& E. Cooke \& F. Jahanian. (2008)."CloudAV:N-version antivirus in the network cloud" . Processing of the 17th USENIX Security Symposium.

[18]J. Oberheide \& E. Cooke \& F. Jahanian. (2007)."Rethinking antivirus: Executable analysis in the network cloud". Proceedings of the $2^{\text {nd }}$ USENIX workshop on hot topics in security (HOTSEC).

[19] K. Priyadarsini \& V. Balasubramanian \& S. Karthik.(2011).“Cloud Testing as a Service” (IJAEST) INTERNATIONAL JOURNAL OF ADVANCED ENGINEERING SCIENCES AND TECHNOLOGIES Vol No. 6, Issue No. 2, pp173 - 177. 
[20] L. Sweeney. (2002).” k-anonymity:A model for protecting privacy". International Journal of Uncertainty Fuzziness and Knowledge-Based Systems.

[21] H. Turner \& J. White \& J. Reed \& J. Galindo \& A. Porter \& M. Marathe \& A. Vullikanti \& Gokhale.(2012)."Building a Cloud-Based Mobile Application Test bed". Software Testing in the Cloud: Perspectives on an Emerging Discipline pp. 382-403.

[22] Sergiy Vilkomir. "Cloud Testing-A State of The Art" Review.pp 213-221

[23]Mladen.A.Vouk,(2008).“Cloud computing: Issues, research and implementations". ITI 2008 30th International Conference on Information Technology Interfaces, 16(4):31-40.

[24] S. Wang\& X. S. Wang.(2010).In-Device Spatial Cloaking for Mobile User Privacy Assisted by the Cloud. Proceedings of the $11^{\text {th }}$ International Conference on Mobile Data Management (MDM), pp. 381.

[25] B. Wrenn, CISSP, ISSEP. Unisys Secure "Cloud Addressing the Top Threats of Cloud Computing". (white paper).Accessed $25^{\text {th }}$ June.

[26]S. Xiao \& W. Gong.(2010). "Mobility can help: protect user identity with dynamic credential".Proceedings. 11th Int. Conference on Mobile Data Management, MDM 10, Missouri, USA.

[27]H. Zhangwei \& X. Mingjun.(2010).”A Distributed Spatial Cloaking Protocol for Location Privacy", Proceedings of the 2nd International Conference on Networks Security Wireless Communications and Trusted Computing (NSWCTC), vol. 2, pp. 468.

[28]X. Zhang \& J. Schiffman \& S. Gibbs \& A. Kunjithapatham \& S. Jeong.(2009)."Securing elastic applications on mobile devices for cloud computing".Proceedings ACM workshop on Cloud computing security, CCSW '09, Chicago, IL, USA.

[29]Z. Zhou \& D. Huang(2011)."Efficient and secure data storage operations for mobile cloud computing". IACR Cryptology ePrint Archive: 185

[30] T.Vengattaraman, P.Dhavachelvan and R. Baskaran (2010). "A Model of Cloud Based Application Environment for Software Testing”. (IJCSIS) International Journal of Computer Science and Information Security, Vol. 7, No.

[31] Fuyang Peng, Bo Deng, and Chao Qi (2012)"CASTE:A Cloud-Based Automatic Software Test Environment". World Academy of Science, Engineering and Technology 712012

[32]Oleksii starov and Sergiy Vilkomir (2013)."Integrated TaaS Platform for Mobile Development Architecture solutions".
[33] P. Mell and T. Grance, "Draft nist working definition of cloud computing - v15," 21. Aug 2009, 2009.

[34]A.Konstantinidis, C.Costa,G. Larkou and D. Zeinalipour yazti (2012). "Demo: A Programming Cloud Of Smartphones". In Proceedings of $10^{\text {th }}$ international conference on Mobile Systems ,applications, and services, ACM New York,NY, USA,pp-465-466.

[35] Leah Muthoni Riungu, Ossi Taipale, Kari Smolander. (2010)."Sotware Testing as an Online Service: observations From Practice".Third international conference on software testing, Verification, and Validation Workshop.

[36] Peter Gilbert, Byung-Gon Chun, Landon P. Cox, Jaeyeon Jung Automating Privacy Testing of Smartphone Applications.

[37]Jun-fei Huang ,yun- zhan Gong,(2012).Remote Mobile Test System:A Mobile Phone Cloud for Application Testing.

[38] Peter Gilbert \& Byung-Gon,(2011) Chun "Vision: Automated Security Validation of Mobile Apps at App Markets".

[39]Selvam.R\&Dr.V.Kartikeyani.(2011).”Mobile Software Testing-Automated Test Caes Design Strategies. International Journal on computer Science and engineering(IJCSE).

[40]Hughes Systique.’Test automation Tools For mobile application :A Brief Survey".

[41]Alexey Markov.”Software Testing Models Against Information Security Reqirements".

\footnotetext{
About the Authors

Shilpa Bahl holds M.Tech in IT from UIET Kurukshetra University Kurukshetra, B.Tech in Electronics and Communication from Kurukshetra University and is currently pursuing Ph.D. in the area of Mobile Application Testing On cloud against information security from Ansal university, Gurgaon. She is working as an Assistant Professor in department of Computer Science Engineering in KIIT College of Engineering, Gurgaon. She is also a member of Computer Society of India.

Manmohan Chaturvedi is a retired Air Commodore from Indian Air Force with $\mathrm{PhD}$ in Information Security domain from IIT Delhi. He has about 35 years of experience in managing technology for IAF. An alumnus of National Defense College, New Delhi, he has held various appointments dealing with operational and policy dimensions of Information and Communication Technology. He graduated from Delhi College of Engineering and completed post graduation from IIT Delhi. Currently he is a Professor at School of Engineering and Technology, Ansal University with research interests in vulnerability of evolving ICT infrastructure and protection of Critical Information Infrastructure.
} 